\title{
The use of phage-sensitivity patterns for tracing heat labile toxin-positive $\left(\mathrm{LT}^{+}\right)$Escherichia coli
}

\author{
K. A. MONSUR, J. D. CLEMENS, D. A. SACK, A. L. MIAH and Y. A. BEGUM
}

International Centre for Diarrhoeal Disease Research, Bangladesh, GPO Box 128, Dhaka 1000, Bangladesh

\begin{abstract}
Summary. The heat-labile toxin (LT) positive Escherichia coli colonies from 785 stool specimens obtained during a cholera vaccine trial were examined for their phagesensitivity pattern to $31 \mathrm{E}$. coli phages. These specimens originated from 105 index cases and their contacts. Isolates with common phage-sensitivity patterns were grouped and were studied further both serologically and for their plasmid profile. The largest group (42 isolates) belonged to serogroup 078 and the second largest group (19 isolates) belonged to serogroup 06 . There were 23 index cases which had $E$. coli with the same phage-sensitivity pattern as some of their contact strains. The identity of isolates from 16 index cases with strains from their respective contacts could be verified serologically. For the remaining seven index cases and their contacts, the isolates did not agglutinate with available antisera. However, subsequent studies demonstrated that, when the phage-sensitivity pattern among the strains matched, the plasmid profiles of these strains also matched. This further indicates the ability of phage-sensitivity patterns to serve as markers in tracing strains.
\end{abstract}

\section{Introduction}

The Escherichia coli population in the intestine of man and animals is a continually changing mixture of many different strains (Sears et al., 1950; Sears and Brownlee, 1952; Wood, 1955; Cruickshank et al., 1973). This turnover should be taken into account in epidemiological investigations of $E$. coli infections. However, such studies have been hampered because there is no routine and easy way to determine the identity of $E$. coli isolates. Consequently, epidemiological studies of $E$. coli infections have been limited by the inability to follow an individual strain from person to person. Serotyping can identify different strains, but this is laborious, time-consuming and beyond the capacity of many laboratories. There is, therefore, a need to develop a practical technique by which $E$. coli can be traced from one individual to another.

Earlier studies indicated that $E$. coli phages may be used as markers for $E$. coli isolates (Smith and Crabb, 1956; Brown and Parisi, 1966; Pande et al., 1976). A bacteriophage $\Omega 8$, specific for $E$. coli serogroup O8, has been isolated (Jann et al., 1971; Wallenfels and Jann, 1974). Gross et al. (1977) isolated a phage specific for the $\mathrm{K} 1$ polysaccharide antigen of E. coli. Gupta et al. (1982) have described

Received 25 May 1988; revised version accepted 26 July 1988. a phage specific for the $\mathrm{K} 5$ antigen. With a group of such phages, it may be possible to identify specific $E$. coli by their phage-sensitivity pattern and some of these patterns may be related to certain virulence patterns of clinical or epidemiological importance.

We have isolated several $E$. coli phages, each of which has a different host range with a variety of $E$. coli strains. When $E$. coli strains are tested against a battery of these selected phages, $>90 \%$ of strains are found to be sensitive to some of the phages and insensitive to others. The net result is that each strain has a unique phage-sensitivity pattern. Thus, if two $E$. coli isolates belong to the same type, their phage-sensitivity pattern will also be the same. The phage-sensitivity pattern can be used as a marker to trace specific $E$. coli strains. In a preliminary study with 24 such phages, this method of $E$. coli identification was demonstrated (Monsur et al., 1985). Using phages, we have evaluated also the pooling method, normally used for detecting enterotoxigenic $E$. coli, by determining the number of each of the original $E$. coli pool members which survived in the pool (Monsur et al., 1986). This report describes the usefulness of phage typing in tracing heat-labile toxin-positive $E$. coli ( $\mathrm{LT}^{+}$ETEC) strains from index case to family contacts in a field situation. 


\section{Materials and methods}

\section{Sources of strains}

The E. coli strains were from 785 faecal samples of patients attending the Matlab Diarrhoea Treatment Centre at the International Centre for Diarrhoeal Disease Research, Bangladesh (ICDDR, B) and their family or neighbourhood contacts. The strains were isolated between June 1985 and April 1986 during a field trial of two new, oral, cholera vaccines (Clemens et al., 1986). Surveillance was simultaneously carried out for diarrhoea resulting from enterotoxigenic $E$. coli (ETEC) as well as cholera, because of the known immunological cross reactivity between the heat labile enterotoxin of $E$. coli (LT) with cholera toxin (CT). When diarrhoea patients with $\mathrm{LT}^{+}$ETEC were identified at the treatment centre, field workers visited the families and neighbours of these index cases to determine if there were other cases of diarrhoea. Active surveillance for diarrhoea symptoms as well as faecal bacteriology was carried out daily for 7 days in each of the family members and neighbours studied.

\section{Bacteriological studies}

All 785 faecal specimens from diarrhoea patients and their contacts were plated on MacConkey Agar (Difco Laboratories, MI, USA) for detection of $\mathrm{LT}^{+}$ETEC. Three typical $E$. coli colonies from each specimen were preserved on trypticase soya agar slants and later assayed for production of LT by its effect on adrenal cells in miniculture (Sack and Sack, 1975). In all, 1350 colonies were identified as LT-producers, of which 120 were from index cases and 1230 from contacts. All of them were tested for their phage-sensitivity patterns with 31 phages; 24 of the phages were those described earlier (Monsur et al., 1985); seven other phages were added with the hope of increasing the sensitivity of the identification. These seven were isolated by the same methods as were the 24 previously used. The tests were performed as described earlier (Monsur et al., 1985) with phages at a concentration of one hundred times the Routine Test Dilution. The tests were carried out approximately one year after collection.

\section{Grouping of isolates}

Isolates were considered to be identical and grouped together when they had the same phage-sensitivity patterns. This matching was performed blindly without any knowledge of the source of the isolates. When an individual had more than one colony of the same phagesensitivity pattern, or when the same phage-sensitivity pattern was detected from an individual on different dates, these isolates were considered to belong to the same strain. Judgement was used in interpreting odd or weak sensitivities, e.g., when the sensitivity pattern of two isolates matched against large number of phages but differed with only one, this difference was ignored.
Sensitivities showing less than ten clear plaques against a phage or when the phage gave minute, indistinct plaques against an isolate were considered to be weak reactions and were normally ignored unless clearly complementary to an obvious interpretation.

\section{Serology}

E. coli colonies were grown overnight at $37^{\circ} \mathrm{C}$ on sheepblood agar plates. Growth was removed and suspended in saline, boiled for $1 \mathrm{~h}$, centrifuged at $3000 \mathrm{~g}$ for $15 \mathrm{~min}$ and the sediment resuspended in saline. Serogrouping was done by standard techniques (Edward and Ewing, 1972 ) with commercially prepared $E$. coli $O$ antisera (Difco Laboratories, Detroit, MI, USA). H serotyping was not performed.

\section{Plasmid profile}

In selected cases the plasmid profiles of the isolates were determined by the method of Birnboim and Doly (1979) for plasmid extraction and by the method of Meyers et al. (1976) for agarose gel electrophoresis.

\section{Results}

\section{Phage sensitivity of bacterial isolates}

Bacteria isolated from 105 index cases (120 colonies) and their 680 contacts (1230 colonies) were collected. Of the 1350 colonies, 1036 ( 85 from index and 951 from contact cases) had unique phage-sensitivity patterns, which did not match with that of any others in the group. They were not studied further. The remaining 314 colonies (35 from index and 279 from contact cases) belonged to a phage-sensitivity pattern, which was common to two or more of the isolates; these were studied further. The major groups of isolates are described below.

1. The largest group, consisting of 42 isolates, included 11 index cases and 17 of their contacts. They all shared susceptibility to the same five phages (table - examples 1.1 and 1.2). All the members of this group were found to belong to serogroup 078. Some members of this group were also sensitive to one or two other phages (tableexample 1.2). In this case the sensitivity to additional phages did not seem to affect the serogroup (table-examples 1.1 and 1.2). There were exceptions when the strains were sensitive to specific phages as will be seen by comparing examples 1.2 and 2 in which the additional sensitivities to two phages (nos 5 and 21) appear to change the serogroup $\mathrm{O} 78$ to $\mathrm{O6}$. In spite of these differences between the members of this group the 
Table. Escherichia coli isolates with similar phage pattern in index and contact isolates-verification of identity by serotyping and plasmid profile.

\begin{tabular}{|c|c|c|c|c|c|c|c|c|c|c|c|c|c|c|c|c|c|c|c|c|c|}
\hline \multirow{2}{*}{$\begin{array}{l}\text { Exam- } \\
\text { ple } \\
\text { no. }\end{array}$} & \multirow{2}{*}{$\begin{array}{l}\text { Specimens* } \\
\text { from }\end{array}$} & \multicolumn{18}{|c|}{ Isolate sensitive $\nmid$ to phage nos. } & \multirow[b]{2}{*}{ Sero-group } & \multirow[b]{2}{*}{ Sizes of plasmids present $(\mathrm{kb})$} \\
\hline & & 2 & 5 & 7 & 8 & 9 & 11 & 13 & 14 & 15 & 16 & 18 & 19 & 21 & 22 & 23 & 28 & 30 & 31 & & \\
\hline 1. & $\begin{array}{l}\text { Group } \\
\text { pattern }\end{array}$ & - & - & - & - & - & - & + & + & + & - & - & - & - & - & + & - & - & + & & \\
\hline \multirow[t]{2}{*}{1.1} & p-48 Index & - & - & - & - & - & - & + & + & + & - & - & - & - & - & + & - & - & + & O78 & $75,10 \cdot 4,7 \cdot 2,4 \cdot 5$ \\
\hline & $\mathrm{p}-48 \mathrm{HCl}$ & - & - & - & - & - & - & + & + & + & - & - & - & - & - & + & - & - & + & O78 & $75,10 \cdot 4,7 \cdot 2,4 \cdot 6$ \\
\hline \multirow[t]{2}{*}{1.2} & p-93 Index & - & - & - & - & - & - & + & + & + & - & - & - & - & - & + & + & - & + & O78 & $95,6 \cdot 5$ \\
\hline & p-93 HC8 & - & - & - & - & - & - & + & + & + & - & - & - & - & - & + & + & - & + & O78 & $95,6 \cdot 5$ \\
\hline 2. & $\begin{array}{l}\text { Group } \\
\text { pattern }\end{array}$ & - & + & - & - & - & - & + & + & + & - & - & - & + & - & + & + & - & + & & \\
\hline \multirow[t]{2}{*}{2.1} & p-44 Index & - & \pm & - & - & - & - & + & \pm & + & - & - & - & + & - & \pm & + & \pm & \pm & O6 & $122,81,39,6 \cdot 1,5 \cdot 4$ \\
\hline & $\mathrm{p}-44 \mathrm{HCl}$ & - & \pm & - & - & - & - & + & \pm & + & - & - & - & + & - & \pm & + & & \pm & O6 & $122,81,39,6 \cdot 1,5 \cdot 4$ \\
\hline \multirow[t]{2}{*}{2.2} & p-37 Index & \pm & + & - & - & - & - & + & $\frac{1}{+}$ & + & + & - & - & + & + & + & + & - & \pm & O6 & $75,51,11 \cdot 6,9 \cdot 6$ \\
\hline & p-37 HC5 & \pm & + & - & - & - & - & + & + & + & + & - & - & + & + & + & + & + & $\bar{t}$ & O6 & $75,51,11 \cdot 6,9 \cdot 6$ \\
\hline \multirow[t]{2}{*}{3.} & p-97 Index & + & - & \pm & + & - & \pm & + & + & - & \pm & + & \pm & + & + & - & + & + & - & O6 & $113,75,55,35,3 \cdot 8,3 \cdot 5$ \\
\hline & $\mathrm{p}-97 \mathrm{NC4}$ & + & - & $\overline{-}$ & + & - & \pm & + & + & - & \pm & + & $\overline{ \pm}$ & + & + & - & + & + & - & O6 & $113,75,55,35,3 \cdot 8,3 \cdot 5$ \\
\hline \multirow[t]{2}{*}{4.} & p-33 Index & - & - & - & + & - & \pm & - & - & - & - & - & + & - & - & - & - & - & - & $\mathrm{O} 8$ & $104,56,9 \cdot 5,4 \cdot 0,3 \cdot 5$ \\
\hline & p-33 HC2 & - & - & - & + & - & \pm & - & - & - & - & - & + & - & - & - & - & - & - & $\mathrm{O} 8$ & $104,56,9 \cdot 5,4 \cdot 0,3 \cdot 5$ \\
\hline \multirow[t]{3}{*}{5.} & p-71 Index & + & - & + & + & + & + & - & - & - & - & - & - & - & - & - & - & - & - & Untypeable & $145,49,14 \cdot 6,1 \cdot 6$ \\
\hline & $\mathrm{p}-71 \mathrm{NC2}$ & + & - & + & + & + & + & - & - & - & - & - & - & - & - & - & - & - & - & Untypeable & $145,49,14 \cdot 6,3 \cdot 5,3 \cdot 1,1 \cdot 6$ \\
\hline & $\mathrm{p}-71 \mathrm{NC} 14$ & + & - & + & + & + & + & - & - & - & - & - & - & - & - & - & - & - & - & Untypeable & $145,49,14 \cdot 6,3 \cdot 5,3 \cdot 1,1 \cdot 6$ \\
\hline
\end{tabular}

${ }^{*} \mathrm{p}-48, \mathrm{p}-93$, etc. $=$ Patient number for the index cases; $\mathrm{HCl}, \mathrm{NC} 2$, etc. $=$ household and neighbour contact numbers etc. for the respective index case.

$\dagger+=$ Fully sensitive strain; $\pm=$ weakly sensitive strain.

phage-sensitivity patterns between isolates from the index cases and their respective contacts matched closely (table-examples 1.1 and 1.2). This indicates that the phage-sensitivity pattern of both the index and contact strains showed the same deviation from the standard (hypothetical) phagesensitivity pattern for the serogroup O78 (tableexample 1.2). Two isolates with borderline sensitivity to this group of phages were found to belong to serogroup $\mathrm{O} 114$ : K90. Of these one was only weakly sensitive to phage nos. 14 and 15 and the other to nos. 14,15 and 31 of the five phages (nos. 13, 14, 15,23 and 31 ) to which members of the 078 group are normally fully sensitive (table).

2. The next group of 19 , and another two small groups of three and two isolates respectively, belonged to serogroup O6. These three groups included three index cases with one or two contact(s) for each index case. Isolates from two of these index cases belonged to the major group (table-examples 2.1 , and 2.2) and the remaining one to a minor group (table - example 3 ). In each case the isolates from the index and contact cases matched closely with each other. Members of the major group, comprising 19 isolates, were sensitive to three phages additional to the five phages to which members of the $\mathrm{O} 78$ group are sensitive (tableexample 2.1). For isolates in this group, sensitivity to one or more additional phages also did not seem to affect the serogroup as long as the basic sensitivity pattern for the group, as shown by example 2, remained intact. There were wide differences in phage-sensitivity patterns between this largest and the other two groups, all belonging to serogroup $\mathrm{O6}$ (table-examples 2.1, 2.2 and 3).

Serogroup O8, comprising 19 isolates, included different, heterogeneous groups of phage-sensitivity patterns and had two index cases with one contact for each index case (table-example 4). The phagesensitivity patterns of the two groups of index cases and their respective contacts differed from each other but the phage-sensitivity pattern for each index and its own contact matched.

The other grouped isolates comprised a collection of heterogeneous phage-sensitivity patterns. In all, those which could be serogrouped accounted for 
111 isolates, which included $42,24,19,13,7,4$, and 2 isolates representing serogroups $078,06,08$, $\mathrm{O} 25, \mathrm{O} 114: \mathrm{K} 90, \mathrm{O} 75$, and $\mathrm{O} 15$ respectively.

\section{Correlation of phage sensitivity with plasmid profile}

On seven occasions the phage-sensitivity patterns of the isolates from index cases matched those of isolates from one or more contacts, but serological verification was not possible with the available antisera. In each of these cases, plasmid profiles of isolates with similar phage-sensitivity patterns also matched (table - example 5).

There were 23 index cases with one or more contacts that harboured $E$. coli with the same phage pattern. These index cases and their contacts accounted for 58 cases. In 16 of the index cases the identity of the isolates from the index case and respective contacts, accounting for a total of 39 casès, could be verified serologically. For the remaining seven index cases and their contacts, a total of 19 cases, in which the isolates did not agglutinate with available antisera, plasmid profile studies showed that when the phage-sensitivity pattern matched, the plasmid profile also matched, (table - example 5), further documenting that isolates which were identical by phage typing were also identical by another criterion. Thus, in all the 58 cases, the phage-sensitivity pattern appeared to be a marker which correctly identified specific strains from contacts and index cases.

\section{Discussion}

The phage-sensitivity pattern is not expected to identify any specific charcteristic of $E$. coli, such as serotype, toxigenicity, etc. Its main value is of a statistical nature based on the unlikely event that two different isolates would have the same phagesensitivity pattern unless the isolates were of the same strain.

When a strain with a particular serotype has a

\section{REFERENCES}

Birnboim H C, Doly J 1979 A rapid alkaline extraction procedure for screening recombinant plasmid DNA. Nucleic Acids Research 7: 1513-1523.

Brown W J, Parisi T J 1966 Bacteriophage typing of bacteriuric Escherichia coli. Proceedings of the Society for Experimental Biology and Medicine (New York) 121 : 259-262.

Clemens J D et al. 1986 Field trial of oral cholera vaccine in Bangladesh. Lancet 2: 124-127.

Cruickshank R, Duguid J P, Marmion B P, Swain R H A 1973 Medical Microbiology. 12th edn, vol 1. Churchill Livingstone Edinburgh, pp 328. unique phage-sensitivity pattern, that pattern may be used to predict the serotype. Further studies should confirm whether the phage-sensitivity pattern, as observed for the serogroup 078 in the present study, may be used to identify unknown $E$. coli belonging to serogroup $\mathrm{O} 78$. The fact that all of the 42 isolates of serogroup 078 had the same standard phage-sensitivity pattern makes it likely that in this pattern we may have a marker which will identify at least most of the heat-labile toxigenic isolates of this serogroup. Similarly because 19 out of 24 of the O6 serogroup have been found to have a unique phage-sensitivity pattern it may also be possible that we have detected a specific phagesensitivity pattern which will identify a substantial number of heat-labile toxigenic O6 serogroup. None of the other serogroups have been found to show a unique phage-sensitivity pattern.

These tests have been performed on isolates which are one year old. Whether tests with fresh isolates and non-toxigenic isolates of $\mathrm{O} 78$ and $\mathrm{O6}$ serogroups will show a difference in pattern remains to be seen.

In the present study, the phage-sensitivity patterns of 42 isolates of serogroup 078 and of 19 isolates of serogroup 06 were sufficiently distinctive to indicate the serogroup. This could then be confirmed by serological testing. However, there were 19 isolates of serogroup $\mathrm{O} 8$ for which no specific phage-sensitivity pattern could be identified. If, among the 31 phages used in the test, we can include phage $\Omega 8$, specific for serogroup 08 (Jann et al., 1971; Wallenfels and Jann, 1974) and also some of the other phages that detect specific $E$. coli antigens (Gross et al., 1977; Gupta et al., 1982), they, together, may be able to identify over $50 \%$ of the serogroups involved in locally prevailing $\mathrm{LT}^{+}$ ETEC infection.

We thank Professor R. Eeckels, Director, ICDDR, B, for his constructive comments in the preparation of this manuscript. This study was funded from a UNDP/WHO grant to ICDDR,B.

Edward P R and Ewing W H 1972 Identification of Enterobacteriaceae, 3rd edn. Burgess, Minneapolis.

Gross R J, Cheasty T, Rowe B 1977 Isolation of bacteriophages specific for the $\mathrm{K} 1$ polysaccharide antigen of Escherichia coli. Journal of Clinical Microbiology 6 : 548-550.

Gupta D S et al. 1982 Coliphage K5, specific for E. coli exhibiting the capsular K5 antigen. FEMS Microbiology Letters 14: 75-78.

Jann K, Schmidt G, Wallenfels B, Freund-Molbert E 1971 Isolation and characterization of Escherichia coli bacteriophage $\Omega 8$ specific for $E$. coli strains belonging to sero-group O8. Journal of General Microbiology 67: 289-297.

Meyers J A, Sanchez D, Elwell L P, Falkow S 1976 Simple 
agarose gel electrophoretic method for the identification and characterization of plasmid deoxyribonucleic acid. Journal of Bacteriology 127: 1529-1537.

Monsur K A, Kay B A, Miah A L, Begum Y A 1986 An evaluation of the pooling method for detecting enterotoxigenic Escherichia coli. Journal of Diarrhoeal Disease Research 4: $211-215$.

Monsur K A et al. 1985 Use of bacteriophage as a marker for identification of freshly-isolated individual Escherichia coli strains. Journal of Diarrhoeal Disease Research 3: 131-137.

Pande R C, Rajvanshi V S, Mehrotra T N, Gupta S C 1976 Phage typing of enteropathogenic serotypes of Escherichia coli from sporadic cases of acute and chronic diarrhoea and gastroenteritis in Allahabad. Indian Journal of Medical Research 64: 841-846.

Sack D A, Sack R B 1975 Test for enterotoxigenic Escherichia coli using $\mathrm{Y} 1$ adrenal cells in miniculture. Infection and Immunity 11 : 334-336.
Sears H J, Brownlee I 1952 Further observations on the persistence of individual strains of Escherichia coli in the intestinal tract of man. Journal of Bacteriology 63 : 47-57.

Sears H J, Brownlee I, Uchiyama J K 1950 Persistence of individual strains of Escherichia coli in the intestinal tract of man. Journal of Bacteriology 59 : 293-301.

Smith H W, Crabb W E 1956 The typing of Escherichia coli by bacteriophage: its application in the study of the $E$. coli population of the intestinal tract of healthy calves and of calves suffering from white scours. Journal of General Microbiology 15: 556-574.

Wallenfels B, Jann K 1974 The action of bacteriophage $\Omega 8$ on two strains of Escherichia coli O8. Journal of General Microbiology 81 : 131-144.

Wood P C 1955 The epidemiology of white scours among calves kept under experimental conditions. Journal of Pathology and Bacteriology 70: 179-193. 